\title{
A clinical perspective on escalating or de-escalating adjuvant therapy in HER2+ breast cancer
}

\author{
Angela Esposito ${ }^{\mathrm{a}}$, Giulia Viale ${ }^{\mathrm{a}, \mathrm{b}}$, Carmen Criscitiello and Giuseppe Curigliano ${ }^{\mathrm{a}, \mathrm{b}}$
}

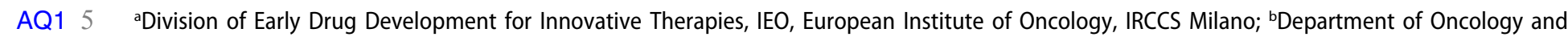
Hemato-Oncology, University of Milano

ABSTRACT

Introduction: Patients with early HER2-positive breast cancer (BC) benefit from HER2-targeted systemic therapy. The endorsed standard adjuvant treatment for patients with early HER2-positive breast cancer is chemotherapy plus trastuzumab administered for 1 year.

Areas covered: Several trials have investigated modifications of the standard treatment in terms of deescalation by either shortening the duration or giving less resource-demanding regimens and in terms of escalation by either adding a second anti-HER2 agent or extending the duration of HER2-targeted treatment for more than 12 months. In this perspective, we would offer a comprehensive view of these trials and discuss their findings.

Expert commentary: At the current state of knowledge, there are still open questions regarding the management of HER2+ BC patients, such as the most adequate duration of trastuzumab therapy, the optimal chemotherapy regimen that should be combined with trastuzumab, and the addition of a second anti-HER2 agent. Growing evidences suggest that some HER2+ BC patients may not need chemotherapy. If these patients could be recognized upfront, optimal response could potentially be reached with HER2-targeted therapy alone.
ARTICLE HISTORY

Received 24 September 2018

Accepted 20 November 2018

\section{KEYWORDS}

Adjuvant trastuzumab duration; breast cancer; HER2-positive breast cancer; HER2-targeted therapy

\section{Introduction}

The human epidermal growth factor receptor 2 (HER2) has been shown to be overexpressed in $15 \%$ of breast cancers (BC) [1]. Overexpression of HER2 in BC seems to confer a more aggressive phenotype and, historically, was correlated with a poor prognosis with lower disease-free survival (DFS) and overall survival (OS) rates, higher risk of recurrence and greater resistance to therapy [2-4]. Patients with early HER2-positive BC benefit from HER2-targeted systemic therapy. The endorsed standard adjuvant treatment for patients with early HER2-positive BC is chemotherapy plus trastuzumab administered for 1 year.

Trastuzumab has dramatically changed the natural course of HER2 positive BC, transforming an aggressive subtype of breast cancer in one that may achieve an excellent prognosis [5-8]. Adjuvant trastuzumab was first investigated in four large randomized trials: the National Surgical Adjuvant Breast and Bowel Project (NSABP) trial B-31, the North Central Cancer Treatment Group (NCCTG) trial N9831, the HERceptin Adjuvant trial (HERA), and the Breast Cancer International Research Group (BCIRG) trial 006 [9-11]. The same trastuzumab dose and the same duration (12 months) were selected for evaluation in all four trials; however, the original decision to give trastuzumab for 1 year was arbitrary and not supported by preclinical nor clinical data [12]. In 2006, the Food and Drug administration (FDA) approved the use of trastuzumab in the adjuvant setting of BC. In the absence of data from other schedules, the 1 year duration became the standard. Updated results of the adjuvant trastuzumab trials, with 8 years [13] and 10 years of median follow up, showed consistent disease-free survival (DFS) and overall survival (OS) benefits with the addition of trastuzumab to chemotherapy $[14,15]$. These data were particularly relevant because investigators of all trials, with the exception of BCIRG006, allowed crossover to trastuzumab for patients enrolled in the control groups of the studies. In addition, the HERA trial has confirmed that 24 months treatment does not further improve DFS and OS [16].The current standard treatment is costly, lengthy and occasionally associated with cardiac adverse events, which occurred in less than $3 \%$ of patients treated in the pivotal clinical trials. In the last years, other studies investigated modifications of the schedule of treatment with trastuzumab by either making it shorter and less toxic (de-escalation), or more effective with dual HER2 inhibition or extended treatment duration (escalation) (Table 1).

\section{De-escalation trials}

Seven randomized trials address the topic whether a shorter regimen of adjuvant trastuzumab may be as effective as 1-year of trastuzumab, but with fewer side-effects.

In four of these trials, trastuzumab is given concomitantly with chemotherapy in the experimental arm with the aim to investigate drug synergism (FinHer, E2198, SOLD, and Short-HER trials), and 
Table 1. Summary of results of major trials addressing the lenght of adjuvant trastuzumab.

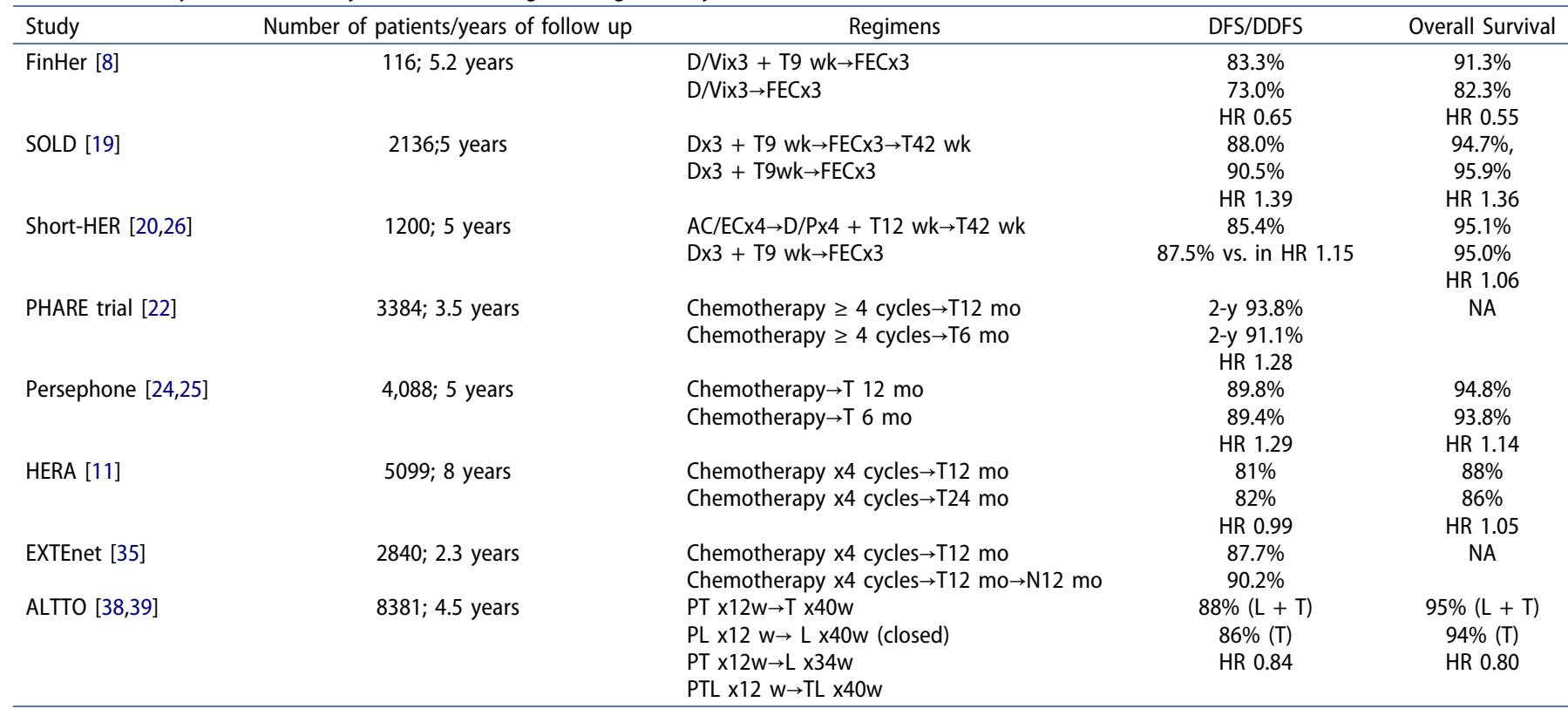

DFS: disease-free survival; T: trastuzumab; D: docetaxel; P:paclitaxel;Vi: vinorelbine; L: lapatinib; FEC; fluorouracil, epirubicin, cyclophosphamide; HR: hazard ratio; wP: weekly paclitaxel; AC: doxorubicin plus cyclophosphamide; N.A.: not available; EC: epirubicin plus cyclophosphamide; P: paclitaxel; N:neratinib,

75 three trials compare 6-month to 12-month duration of trastuzumab (the Hellenic trial, PHARE, and PERSEPHONE) [8,17-25].

In the FinHer study [8], 1010 women with axillary nodepositive or high-risk node-negative breast cancer were randomized to receive three cycles of docetaxel or vinorelbine, followed in both groups by three cycles of fluorouracil (F), epirubicin (E), and cyclophosphamide (C). Patients with HER2positive breast cancer $(n=232)$ were further treated with trastuzumab or no additional therapy. Even with the shorter duration of trastuzumab after a median follow-up of 8 years, distant DFS (83.3\% vs $73 \%$ ) and OS (91.3\% vs $82.3 \%$ ) favored the trastuzumab arm, but the difference in OS was not statistically significant. The trial showed a benefit, although this was a small trial, and the docetaxel dose was $100 \mathrm{mg} / \mathrm{m} 2$ in about a half of patients, while now many patients receive a smaller dose [8].

In SOLD trial [19], 2,176 patients with early-stage HER2positive $B C$ were randomized (1:1) to the 9-week trastuzumab arm or the 12-month trastuzumab arm. Patients in both arms received three cycles of docetaxel $(80 \mathrm{mg} / \mathrm{m} 2$ or $100 \mathrm{mg} / \mathrm{m} 2)$ and trastuzumab three times a week, followed by three cycles of chemotherapy. Patients in the 9-week arm received no further treatment, whereas those in the 12-month arm received trastuzumab every 3 weeks for 14 cycles. The trial failed to demonstrate that 9 weeks of adjuvant trastuzumab were not inferior to the standard 12 months in terms of DFS

100 [19].The 5-years estimated DFS rate was $90.5 \%$ with 1 year of trastuzumab versus $88.0 \%$ with 9 weeks (HR, 1.39; $90 \% \mathrm{Cl}$, 1.12-1.72). The 5-year estimated OS rates were $95.9 \%$ versus $94.7 \%$, respectively ( $\mathrm{HR}, 1.36 ; 90 \% \mathrm{Cl}, 0.98-1.89)$. The estimated 5-year rate of patients without distant recurrence (dis105 tant-free survival) was also higher with longer trastuzumab at $94.2 \%$ compared with $93.2 \%$, respectively $(\mathrm{HR}, 1.24 ; 90 \% \mathrm{Cl}$, 0.93-1.65).The shorter trastuzumab treatment was safer to the heart than the longer treatment. In the 9-week group there have been 22 protocol-defined cardiac adverse events compared with 42 in patients receiving 1 year of trastuzumab $(p=.012)$. Congestive heart failure occurred in 21 and 36 patients in the 2 arms, respectively $(p=.046)$. Due to cancer characteristics (many patients had node-negative cancer and some investigators preferred not to enroll patients with a high risk of cancer recurrence) and some logistical issues, the planned number of DFS events was not reached within a reasonable period; hence, the study had lower statistical power than planned, thus limiting the interpretation of the results.

In the Short-HER study $[20,26]$, patients were randomly selected to receive 1 year of trastuzumab plus chemotherapy ('long' group) or 9 weeks of trastuzumab plus chemotherapy ('short' group). The primary endpoints were DFS and OS. Secondary endpoints included failure rate at 2 years and the incidence of cardiac events. The 5-year DFS did was not noninferior in the frequentist analysis $(87.5 \%$ vs. $85.4 \%$ in the long and short groups, respectively, hazard ratio [HR] 1.15, $90 \% \mathrm{Cl}$ $[0.91,1.46])$. In an analysis of DFS in patients with earlier-stage disease (stage I and II) as compared to those with locally advanced disease (stage III), the shortened duration was not inferior to the longer one. There was no difference in OS at 5 years. There was an ongoing decline in left ventricular ejection fraction for the long group. Therefore, patients with stage III disease and multiple positive lymph nodes may derive greater benefit from a longer duration of trastuzumab, while a selected group of patients who cannot tolerate 12 months of therapy may be reasonably treated with a shorter duration [26]. The openlabel randomized phase III PHARE trial [22] randomized 3384 patients with HER2-positive early BC who had received at least four cycles of chemotherapy and up to 6 months of adjuvant trastuzumab to either continue trastuzumab for another 6 months or to stop trastuzumab at 6 months. After 3.5 years of follow-up, 175 DFS events occurred in patients assigned to 12 months of trastuzumab compared with 219 events in the 
145 6-month group. Data indicated that the 2-year DFS was 93.8\% for patients assigned to the standard of care as compared to $91.1 \%$ for the 6-month regimen $(\mathrm{HR}=1.28 ; 95 \% \mathrm{Cl}, 1.01-1.56 ; p=.29)$. A greater percentage of patients assigned to 12 months of treatment experienced cardiac events compared with patients assigned to 6-month treatment $(5.7 \%$ of patients vs $1.9 \%$; $p<.0001)$. Despite the increased rate of cardiac events that occurred in patients assigned to 12-month treatment, the researchers concluded that the longer regimen should remain the standard of care [22].

Persephone is a randomized phase III non-inferiority trial comparing 6 months of trastuzumab to the standard 12 months in 4,088 patients enrolled from 152 sites in the United Kingdom between 2007 and 2015 [24,25]. Patients received standard chemotherapy regimens as per institutional practice as either adjuvant chemotherapy or neoadjuvant chemotherapy, and either concurrently with or sequentially to trastuzumab, and trastuzumab for either 6 or 12 months based on random allocation. Randomization occurred before the 10th cycle of trastuzumab. At a median followup of 5 years, the researchers found near-identical results between the two treatment arms: DFS was $89.4 \%$ among women in the 6-month arm and $89.8 \%$ among women in the 12-month arm (hazard ratio, 1.29). Only $4 \%$ of women who received trastuzumab for 6 months stopped treatment early due to heart problems, compared to $8 \%$ of those who received trastuzumab for 12 months. The study results demonstrate that 6 months of trastuzumab is non-inferior to 12 months. A pre-defined sub group analysis revealed that groups more likely to benefit from 12 months of trastuzumab therapy compared to 6 months included those patients who received trastuzumab concurrently with chemotherapy, patients who received neoadjuvant chemotherapy, those who received taxanes and among patients who had ER negative disease. The investigators emphasized that-although a few other studies have evaluated shorter durations of trastuzumabPersephone is the largest non-inferiority trial. Ongoing research is evaluating quality-of-life and patient-reported outcomes in this population. Important translational research will be carried out analyzing blood and tumor samples to look for biomarkers to identify subgroups of different risk where shorter/longer durations of trastuzumab might be tailored.

Besides the duration and timing of trastuzumab therapy, the drugs combined to trastuzumab changed across the trials. Hypothetically, the duration of trastuzumab administration might become relatively less meaningful as the efficacy of the drug combinations augments.

The timing of randomization also varied. While in the FinHer, E2198, SOLD, and the Hellenic trial randomization occurred prior to beginning systemic therapy; randomization was done after finishing chemotherapy in PHARE, and prior to the 10th trastuzumab cycle in PERSEPHONE. The timing of randomization may affect the study patient populations, as patients who progress rapidly or who do not tolerate therapy are excluded when randomization is done after chemotherapy.

Besides shortening duration of adjuvant treatment, deescalation strategies also include a reduction of chemotherapy or the use of less toxic agents instead of chemotherapy.

The BCIRG006 trial $[9,14]$ of the Breast Cancer International Research Group evaluated a non- anthracycline regimen with docetaxel and carboplatin plus trastuzumab for a total of 52 weeks ( $\mathrm{TCH}$ regimen) and compared it to a standard anthracycline and taxane containing chemotherapy \pm trastuzumab ( $A C \rightarrow T$ and $A C-T H$, respectively). The $\mathrm{TCH}$ regimen resulted in significantly fewer cardiac events with a small difference in estimated DFS compared to AC-TH at 10.3 years of follow up (DFS $74 \cdot 6 \%$ vs $73 \%$; OS $85 \cdot 9 \%$ vs $83 \cdot 3 \%$, respectively). The BCIRG006 trial, which included also patients with small tumors ( $40 \%$ were $\mathrm{pT} 1$ ) and no axillary involvement $(28 \%)$, showed the benefit of the addition of trastuzumab to chemotherapy even in a low-risk population; a metanalysis by O'Sullivan et al. confirmed these results [27].

However, in this low-risk population, a possible strategy to spare toxicity might be the reduction of chemotherapy while maintaining trastuzumab administration for one year, as suggested by the APT trial [28]. This single arm prospective study investigated a regimen with weekly paclitaxel and trastuzumab for 12 weeks, followed by 9 months of trastuzumab monotherapy in women with small HER2-positive tumors, predominantly stage I. At 4-year follow-up, DFS was $98.7 \%$, with excellent tolerability. These results are also in line with data reported by a recent observational study from the Netherlands [29]. Some studies are also evaluating to target the HER2 receptor with new drugs and combinations. The phase II ATEMPT trial [30] randomizes patients with stage I HER2-positive breast cancer to receive either T-DM1 or paclitaxel in combination with trastuzumab, followed by 1 year of trastuzumab to evaluate the possible omission of chemotherapy with taxane in this population. In the same direction of de-escalating treatment, the phase II ATOP trial [31] evaluates the role of T-DM1 in treating older patients with stage I-III HER2 positive BC, who decline or are not candidates to standard chemotherapy.

\section{Escalation trials}

A 2008 meta-analysis including HERA, NCCTG-N9831, NSABPB31, BIRCG-006, FinHER, and PACS-04 trials assessed the benefit of adjuvant trastuzumab, showing a mean decrease in risk recurrence of about $37 \%$ and in risk mortality of about $34 \%$ [32]. The question addressed in subsequent clinical trials in this setting is whether we could do any better.

The optimal duration of adjuvant trastuzumab treatment remains 1 year. The HERA trial evaluated length of therapy, comparing an observational arm with no trastuzumab, a 1-year trastuzumab arm and a 2-year trastuzumab arm [11]. At a median 8-year follow-up, no difference in DFS was observed in patients treated with adjuvant trastuzumab for 1 or 2 years ( $\mathrm{HR}, 0.99 ; 95 \% \mathrm{Cl}, 0.85-1.14 ; p=.86)$, while there was an increased rate of grade $3 / 4$ adverse events and higher cardiac toxicity $(4.1 \%$ and $7.2 \%$ for the 1-year and 2-year groups, respectively) [16,33]. Another phase III randomized study, the EXTENET trial, evaluated one year of HER2targeted therapy with neratinib versus placebo in 2840 patients who had completed one year of trastuzumab for early stage disease [34]. In addition, to investigate a new potential effective drug in the adjuvant setting, this study also evaluates 2 years of adjuvant HER2-targeted treatment. A prespecified early analysis at the 2-year mark showed an 
improved DFS in patients receiving neratinib, especially in 260 case of hormone receptor co-expressing cancer(HR 0 - 51, 95\% Cl $0 \cdot 33-0 \cdot 77 ; p=0 \cdot 0013$ ).At 2-year follow-up, invasive DFS was $93.9 \%$ for neratinib arm compared to $91.6 \%$ for the control arm; subsequent data showed a 5-year invasive DFS of $90.2 \%$ in the neratinib group compared to $87.7 \%$ in the placebo group [35]. This benefit was counterbalanced by a severe gastrointestinal toxicity, with $40 \%$ of patients reporting grade 3 diarrhea, leading to dose reductions in $26 \%$ and treatment discontinuation in $17 \%$ of the patients [34]. Notably, at the time of ExteNET study design, management of diarrhoea was instituted only after the development of symptoms. Studies exploring different strategies for gastrointestinal toxicity, including the use of prophylactic loperamide, are under investigation. At SABCS 2017 were presented the initial data of CONTROL trial. In this trial, patients with HER2-positive early stage breast cancer who had completed trastuzumab-based adjuvant therapy received neratinib daily for a period of one year + oral loperamide prophylaxis for one or two cycles (1 cycle $=28$ days) \pm budesonide or colestipol for the first cycle. The results showed that a structured loperamide prophylactic regimen for 1 or 2 cycles reduces the incidence, severity and duration of neratinib-associated diarrhea compared with events observed in the ExteNET trial. Adding budesonide or colestipol appears to further diminish the duration and number of episodes of diarrhea and improves neratinib tolerability [36].Based on the results of ExteNET trial, the FDA approved neratinib for patients with early-stage HER2 positive breast cancer who have finished 1 year of adjuvant trastuzumab. The European Committee for Medicinal Products for Human Use (CHMP) adopted a positive opinion recommending marketing authorization for neratinib in June 2018, after an initial negative opinion; however, it concluded that benefits seemed to be largely confined to patients with hormonereceptor positive cancer [37].

Indeed, given the lack of OS data, the not clinically and statistically significant $1.7 \%$ improvement in distant DFS at 5 years in the entire population, the gastrointestinal toxicity and the cost, extended adjuvant neratinib should not be recommended to all patients. However, it should be discussed, especially in the high-risk population, with locally advanced HER2-positive, estrogen receptor-positive breast cancer, many positive nodes or residual disease after neoadjuvant therapy.

Important amendments were instituted during the course of the trial. With the acknowledgement that patients with node-negative disease have a good prognosis with standard chemotherapy/trastuzumab treatment, eligibility was limited to patients with node-positive disease, but only after 671 patients with node-negative cancer had been enrolled. This represented $24 \%$ of the patients in the final intent-to-treat analysis, and their inclusion may have restricted slightly the difference between the investigational and control groups. Another important amendment was to reduce the allowed interval between completion of trastuzumab and enrollment from 2 years to 1 year, thereby capturing patients who might have experienced a recurrence during the second year off therapy. Given these considerations, a $2.3 \%$ absolute improvement in invasive disease-free survival is evidence of the activity of neratinib in this population, especially in patients with higher risk disease.

Another oral tyrosin kinase inhibitor, lapatinib, has been investigated in the adjuvant setting alone (as in TEACH trial [38]), or in addition to trastuzumab. The ALTTO trial (Adjuvant Lapatinib and/or Trastuzumab Treatment Optimization) $[39,40]$ compared a year of adjuvant trastuzumab to a year of lapatinib, but also evaluated a sequential strategy (12 weeks of trastuzumab followed by 34 weeks of lapatinib) and a combination approach with dual HER2 targeting (trastuzumab plus lapatinib for 52 weeks). The study was conducted between 2007 and 2011 and enrolled 8,381 patients. In 2011, after an interim analysis, the lapatinib monotherapy arm was closed for futility to demonstrate non-inferiority of lapatinib versus trastuzumab. At a protocol-specified analysis with a median follow-up of 4.5 years, the combination of trastuzumab and lapatinib was not shown to significantly improve DFS compared with trastuzumab alone $(\mathrm{HR}, 0.84 ; 95 \% \mathrm{Cl}, 0.70-1.02$; $p=.048)$, nor the sequential strategy provided a benefit over trastuzumab (HR, 0.96; 95\% Cl, 0.80-1.15; $p=.61)$. Moreover, lapatinib was associated with lower rates of completion of HER2-targeted therapy, due to its notable toxicity profile (lapatinib led to higher rates of diarrhea, cutaneous rash, and hepatic toxicity were observed, whereas cardiac toxicity was low in all treatment arms) [40].

The addition of bevacizumab to anthracycline and nonanthracycline ( $\mathrm{TCH}$ ) adjuvant trastuzumab regimens was investigated in the phase III BETH trial [41] This study randomized 3509 patients to receive a total of one year adjuvant trastuzumab alone or in combination to bevacizumab following chemotherapy. At a median follow-up of 38 months, the invasive DFS rates were $92 \%$ for both groups and no significant difference in efficacy between the bevacizumab and nobevacizumab regimens was observed, despite additional toxicity in the bevacizumab arms. Thus, this strategy has not been further explored.

Pertuzumab-a recombinant humanized monoclonal antibody that targets the extracellular dimerization domain of HER2 - has been recently approved by FDA and EMA as adjuvant therapy for early stage HER2 positive high-risk (estrogen receptor negative and node positive) breast cancer in combination with chemotherapy and trastuzumab for one year (up to 18 cycles). The approval was obtained based on the results of the phase III randomized APHINITY trial [42], which evaluated efficacy and safety of the addition of pertuzumab to adjuvant trastuzumab and chemotherapy in 4805 patients with early HER2-positive breast cancer. Patients with tumors smaller than $1 \mathrm{~cm}$ were excluded; overall $63 \%$ of patients had node-positive disease, and $36 \%$ had hormone receptors negative disease. At an early follow-up of 3 years, the rate of invasive DFS was $94.1 \%$ in the pertuzumab group and $93.2 \%$ in the placebo group. When evaluating results by nodal status, the benefit was greater in patients with node-positive disease (invasive- DFS was $92.0 \%$ in the pertuzumab group, as compared with $90.2 \%$ in the placebo group). A treatment effect was most detectable among patients who were at higher risk for hormone-receptor negativity (invasive-DFS was $92.8 \%$ in the pertuzumab group and $91.2 \%$ in the placebo group), but

.


375 the effect was statistically homogeneous throughout all subgroups.

Trastuzumab emtansine (T-DM1) is an antibody-drug conjugate already approved in the metastatic setting that is under evaluation also in early HER2 positive BC. Several trials investigating the role of T-DM1 as adjuvant therapy are ongoing, in addition to the ones already mentioned above. The phase III KATHERINE study investigates the use of adjuvant T-DM1 (compared to trastuzumab) in patients with residual disease after neoadjuvant therapy [43], the data will be presented at the 2018 San Antonio Breast Cancer Symposium. The phase III KAITLIN trial compares adjuvant T-DM1 plus pertuzumab to trastuzumab, pertuzumab, and a taxane (weekly paclitaxel or docetaxel), each given after anthracyclines and the results are still awaited [44].

\section{Conclusions}

Since the development of trastuzumab, several drugs, antibodies, antibody-drug conjugates, and tyrosine kinase inhibitors have become part of the useful arsenal for the treatment of HER2-positive BC. Until now, the overall strategy has escalated the treatment by combining more HER2-targeted agents.

However, the treatment escalation is encumbered by high cost and significant toxicity, and in some cases might be an overtreatment. Therefore, redesigning the current treatment strategies is crucial and de-escalation is a research priority to diminish adverse effects without compromising patient outcome.

\section{Expert commentary}

At the current state of knowledge, there are still open questions regarding the management of HER2+ BC patients, such as the most adequate duration of trastuzumab therapy, the optimal chemotherapy regimen that should be combined with trastuzumab, and the addition of a second anti-HER2 agent. Growing evidences suggest that some HER2+ BC patients may not need chemotherapy [28].

If these patients could be recognized upfront, optimal response could potentially be reached with HER2-targeted therapy alone. On the other hand, tumors that do not take advantage from this approach may be treated with chemotherapy or other strategies to overcome resistance. Translational research is underway to look for biomarkers of risk recurrence, which could help determining the optimal trastuzumab therapy for each patient and identifying different risk groups.

Although many biomarkers are under evaluation, no one is already able to guide patient selection for best trastuzumab therapy. A meta-analysis conducted by Loibl et al. [45] shows a lower rate of pathologic complete response $(p C R)$ in presence of a PIK3CA mutation, which may confer resistance to anti-HER2 therapy; however, this difference in pCR does not translate into a DFS difference. These results suggest that although those patients without a PIK3CA mutation may obtain higher $\mathrm{pCR}$ rates than those with a mutation, the mutation is not predictive of long-term outcomes and thus cannot be informative of patient selection. Also, data on the predictive role of tumor infiltrating lymphocytes (TILs) in patients receiving anti HER2 therapy are discordant $[46,47]$ and further work must be done to evaluate whether patients with high TILs may have similar outcomes with less chemotherapy, and whether substituting chemotherapy with immunotherapy may be favorable for these patients. Consideration of de-escalation therapy is also warranted for the group of patients who obtains a pCR after preoperative therapy. Results of a pooled analysis of 12 trials $(11,955$ patients) showed that patients with HER2-positive breast cancer who achieve a pCR have better long-term outcomes, with improved DFS and OS [48]. However, considering the lack of association between treatment effects and long-term outcomes, randomized trials with long-term follow up are required to understand outcomes for specific therapies. Not less important is the identification of mechanisms of resistance and data from neoadjuvant trials could be useful to discover key determinants of response and resistance to anti HER2 therapy.

\section{Five-year view}

In the next years, it will be necessary a different therapeutic approach based on new predictive tools that allow an accurate identification and stratification of patients according to the risk. The launch of new clinical trials with the aim to select patients on the base of these multi-parameter molecular predictors should be considered in the neoadjuvant setting. Neoadjuvant treatment is currently recommended and frequently used in clinical practice in patients with HER2-positive operable breast cancer. Several trials, including NeoSphere [49], TRYPHAENA [50] and GeparSepto studies [51] showed the benefit of combining chemotherapy with dual anti-HER2 directed treatment with pertuzumab and trastuzumab. This approach has led to increased $\mathrm{pCR}$ rates with no new or long term safety concerns. Of interest, in many neoadjuvant trials, the possibility to de-escalate treatment with chemo-free regimens has been explored. The largest reported trial of dual HER2-targeted neoadjuvant therapy without concurrent chemotherapy is the PAMELA study, in which 151 patients received trastuzumab and lapatinib with possibly concomitant endocrine treatment according to tumor subtype and showed [52]. The chemo-free combination in the PAMELA study led to a $\mathrm{PCR}$ rate of $30 \%$ [52] and similar results were obtained in TBCRC006 [53] and TBCRC023 [54] trials investigating the same regimen. In the NeoSphere trial, 107 patients were treated with trastuzumab and pertuzumab without chemotherapy with some benefit ( $\mathrm{pCR}$ rate $27 \%$ in HER2 positive/hormone receptors negative breast cancer patients), albeit superior results were observed for patients treated with concurrent chemotherapy [49]. Similarly, the addition of taxane monotherapy to dual HER2 blockade substantially increased pCR rates in HER2positive/hormone receptors negative breast cancer patients compared with dual blockade alone in the WSG-ADAPT trial [55]. The possible use of TDM1 plus pertuzumab in the neoadjuvant setting was explored in the KRISTINE/TRIO-021 trial, but again the efficacy was inferior to standard treatment [56]. 
Thus, despite the combination of chemotherapy and dual anti-HER2 directed treatment provided the highest rate of $\mathrm{pCR}$ across trials and still remain the standard of care, nonchemotherapy combinations of HER2-targeted therapy could represent a possible option in those patients with health issues that preclude the use of chemotherapy. However these results further support the convenience to use of neoadjuvant study design to investigate the topic. Indeed, the $\mathrm{pCR}$ rate seems to be a valid surrogate marker of long-term outcome, mainly for HER2495 positive breast cancer [48]. Furthermore, the neoadjuvant setting allows performing serial biopsies and in vivo molecular analyses that are critical to assess for novel markers of response or resistance to therapy. Since neoadjuvant trials are not always powered to evaluate long-term outcomes, adjuvant studies are still 500 needed to validate promising findings from the neoadjuvant setting. In addition, despite the progresses in the treatment of HER2 positive $B C$ many patients still die, calling for the identification of newer and better therapies. It could be interesting testing the combinations with PI3K inhibitors, mTOR-targeting agents, CDK4 and CDK6 inhibitors, or immunotherapies (eg, anti-PD-L1 antibodies), which would increase the likelihood of developing effective treatments.

\section{Key issues}

- The advent of anti-HER2 therapies has changed the prognosis of HER2-overexpressing breast cancer.

- The current standard adjuvant treatment for patients with early HER2-positive breast cancer is chemotherapy plus trastuzumab administered for 1 year but it is costly, lengthy, and occasionally associated with cardiac toxicity.

- Several trials have investigated modifications to the standard treatment: de-escalation and escalation regimens.

- The actual strategy is to escalate treatment by combining more HER2-targeted agents.

However, the treatment escalation is encumbered by high cost and significant toxicity. De-escalation strategy, which can be achieved by either reducing or eliminating chemotherapy, could be useful to mitigate the adverse effects without influencing patient outcome.

\section{Funding}

This paper was not funded.

\section{Declaration of interest}

The authors have no relevant affiliations or financial involvement with any organization or entity with a financial interest in or financial conflict with the subject matter or materials discussed in the manuscript. This includes employment, consultancies, honoraria, stock ownership or options, expert testimony, grants or patents received or pending, or royalties.

\section{Reviewer Disclosures}

A reviewer on this manuscript has disclosed affiliations with Roche and Puma.

\section{References}

Papers of special note have been highlighted as either of interest $(\cdot)$ or of considerable interest $(\cdot \bullet)$ to readers.

1. Slamon DJ, Godolphin W, Jones LA, et al. Studies of the HER-2/neu proto-oncogene in human breast and ovarian cancer. Science. 1989;244:707-712.

2. Sjogren $S$, Inganäs $M$, Lindgren $A$, et al. Prognostic and predictive value of c-erbB-2 overexpression in primary breast cancer, alone and in combination with other prognostic markers. J Clin Oncol. 1998;16:462-469.

3. Gabos Z, Sinha R, Hanson J, et al. Prognostic significance of human epidermal growth factor receptor positivity for the development of brain metastasis after newly diagnosed breast cancer. J Clin Oncol. 2006;24:5658-5663.

4. Dawood S, Broglio K, Buzdar AU, et al. Prognosis of women with metastatic breast cancer by HER2 status and trastuzumab treatment: an institutional-based review. J Clin Oncol. 2010;28:92-98.

5. Musolino A, Ciccolallo L, Panebianco $M$, et al. Multifactorial central nervous system recurrence susceptibility in patients with HER2- positive breast cancer: epidemiological and clinical data from a population-based cancer registry study. Cancer. 2011;117:1837-1846.

6. Gianni L, Eiermann W, Semiglazov V, et al. Neoadjuvant and adjuvant trastuzumab in patients with HER2-positive locally advanced breast cancer (NOAH): follow-up of a randomised controlled superiority trial with a parallel HER2-negative cohort. Lancet Oncol. 2014;15:640-647.

7. von Minckwitz G, Rezai M, Fasching PA, et al. Survival after adding capecitabine and trastuzumab to neoadjuvant anthracycline taxane- based chemotherapy for primary breast cancer (GBG 40geparQuattro). Ann Oncol. 2014;25:81-89.

8. Joensuu H, Bono P, Kataja V, et al. Fluorouracil, epirubicin, and cyclophosphamide with either docetaxel or vinorelbine, with or without trastuzumab, as adjuvant treatments of breast cancer: final results of the FinHer Trial. J Clin Oncol. 2009;27:5685-5692.

9. Slamon D, Eiermann W, Robert N, et al. Adjuvant trastuzumab in HER2-positive breast cancer. N Engl J Med. 2011;365:1273-1283.

.. This trial assessed the rationale and efficacy of adding one year of trastuzumab to adjuvant chemotherapy and showed the benefit of the nonanthracycline $\mathrm{TCH}$ regimen in this setting

10. Romond EH, Perez EA, Bryant J, et al. Trastuzumab plus adjuvant chemotherapy for operable HER2-positive breast cancer. N Engl J Med. 2005;353:1673-1684.

- This trial showed improved outcomes obtained with the combination of trastuzumab and paclitaxel following adjuvant doxorubicin and cyclophosphamide

11. Piccart-Gebhart MJ, Procter M, Leyland-Jones B, et al. Trastuzumab after adjuvant chemotherapy in HER2-positive breast cancer. N Engl J Med. 2005;353:1659-1672.

12. Pinto AC, Ades F, de Azambuja E, et al. Trastuzumab for patients with HER2 positive breast cancer: delivery, duration and combination therapies. Breast. 2013;22(Suppl. 2):S152-5.

13. Perez EA, Romond EH, Suman VJ, et al. Trastuzumab plus adjuvant chemotherapy for human epidermal growth factor receptor 2-positive breast cancer: planned joint analysis of overall survival from NSABP B-31 and NCCTG N9831. J Clin Oncol. 2014;32:3744-3752.

14. Slamon DJ, Eiermann W, Robert NJ, et al. Ten year follow-up of BCIRG-006 comparing doxorubicin plus cyclophosphamide followed by docetaxel $(\mathrm{AC} \rightarrow \mathrm{T})$ with doxorubicin plus cyclophosphamide followed by docetaxel and trastuzumab $(A C \rightarrow T H)$ with docetaxel, carboplatin and trastuzumab (TCH) in HER2+ early breast cancer. Cancer Res. 2015;76(suppl):abstr S5-04.

15. Jackisch C, Piccart MJ, Gelber RD, et al. HERA TRIAL: 10-year follow up of trastuzumab after adjuvant chemotherapy in HER2 positive breast cancer - final analysis. Cancer Res. 2015;76(suppl):abstr PD5-01.

16. Cameron D, Piccart-Gebhart MJ, Gelber RD, et al. 11 years' followup of trastuzumab after adjuvant chemotherapy in HER2-positive early breast cancer: final analysis of the HERceptin adjuvant (HERA) trial. Lancet. 2017;389:1195-2105.
535

540

545 
.- This trials showed no added benefit of an extended 2 years period of adjuvant trastuzumab

17. Joensuu $\mathrm{H}$, Kellokumpu-Lehtinen $\mathrm{PL}$, Bono $\mathrm{P}$, et al. Adjuvant docetaxel or vinorelbine with or without trastuzumab for breast cancer. N Engl J Med. 2006;354:809-820.

18. Schneider BP, O'Neill A, Shen F, et al. Pilot trial of paclitaxel-trastuzumab adjuvant therapy for early stage breast cancer: a trial of the ECOG-ACRIN cancer research group (E2198). Br J Cancer. 2015;113:1651-1657.

19. Joensuu $H$, Fraser J, Wildiers $H$, et al. Effect of adjuvant trastuzumab for a duration of 9 weeks vs 1 year with concomitant chemotherapy for early human epidermal growth factor receptor 2-positive breast cancer: the SOLD randomized clinical trial. JAMA Oncol. 2018;4(9):1199-1206.

20. Guarneri V, Frassoldati A, Bruzzi P, et al. Multicentric, randomized phase III trial of two different adjuvant chemotherapy regimens plus three versus twelve months of trastuzumab in patients with HER2-positive breast cancer (Short-HER Trial;NCT00629278). Clin Breast Cancer. 2008;8:453-456.

21. Mavroudis D, Saloustros E, Malamos N, et al. Six versus 12 months of adjuvant trastuzumab in combination with dose-dense chemotherapy for women with HER2-positive breast cancer: a multicenter randomized study by the Hellenic Oncology Research Group (HORG). Ann Oncol. 2015;26:1333-1340.

22. Pivot $X$, Romieu $G$, Debled $M$, et al. 6 months versus 12 months of adjuvant trastuzumab for patients with HER2-positive early breast cancer (PHARE): a randomised phase 3 trial. Lancet Oncol. 2013;14:741-748.

23. Pivot $X$, Suter T, Nabholtz JM, et al. Cardiac toxicity events in the PHARE trial, an adjuvant trastuzumab randomised phase III study. Eur J Cancer. 2015:51:1660-1666.

24. Earl HM, Cameron DA, Miles D, et al. The PERSEPHONE trial: duration of Trastuzumab with Chemotherapy in women with HER-2 positive early breast cancer. J Clin Oncol. 2012;30(suppl): abstrTPS660. http://meetinglibrary.asco.org/content/97920-114

25. Earl HM, Hiller L, Vallier AL, et al. PERSEPHONE: 6 versus 12 months (m) of adjuvant trastuzumab in patients (pts) with HER2 positive (+) early breast cancer $(E B C)$ : randomised phase 3 non-inferiority trial with definitive 4-year (yr) disease-free survival (DFS) results. J Clin Oncol. 2018;36(suppl):abstr 506.

- This is the largest non-inferiority trial to evaluate a shorter duration of adjuvant trastuzumab

645 26. Conte PF, Bisagni G, Frassoldati A, et al. 9 weeks vs 1 year adjuvant trastuzumab in combination with chemotherapy: results of the phase III multicentric Italian study Short-HER. J Clin Oncol. 2017;35(suppl):abstr 501.

27. O'Sullivan CC, Bradbury I, Campbell C, et al. Efficacy of adjuvant trastuzumab for patients with human epidermal growth factor receptor 2-positive early breast cancer and tumors $\leq 2 \mathrm{~cm}$ : a metaanalysis of the randomized trastuzumab trials. J Clin Oncol. 2015;33:2600-2608.

28. Tolaney SM, Barry WT, Dang CT, et al. Adjuvant paclitaxel and trastuzumab for node-negative, HER2-positive breast cancer. N Engl J Med. 2015;372:134-141.

29. van Ramshorst MS, van der Heijden-van der Loo M, Dackus GMHE, et al. The effect of trastuzumab-based therapy on overall survival in small, node-negative HER2-positive breast cancer: to treat or not to treat? Breast Cancer Res Treat. 2016;158(2):361-371.

30. T-DM1 vs paclitaxel/trastuzumab for breast (ATEMPT Trial). NCT01853748.

31. Trastuzumab emtansine in treating older patients with human epidermal growth factor receptor 2-positive stage I-III breast cancer. NCT02414646.

32. Dahabreh IJ, Linardou $H$, Siannis $F$, et al. Trastuzumab in the adjuvant treatment of early-stage breast cancer: a systematic review and meta-analysis of randomized controlled trials. Oncologist. 2008;13:620-630.

33. Goldhirsch A, Gelber RD, Piccart-Gebhart MJ, et al. Herceptin Adjuvant (HERA) trial study team. 2 years versus 1 year of adjuvant trastuzumab for HER2-positive breast cancer (HERA): an open-label, randomised controlled trial. Lancet. 2013;382:1021-1028.

34. Chan A, Delaloge S, Holmes FA, et al. ExteNET study group. neratinib after trastuzumab-based adjuvant therapy in patients with HER2- positive breast cancer (ExteNET): a multicentre, randomised, double- blind, placebo-controlled, phase 3 trial. Lancet Oncol. 2016;17:367-377.

.. This study evaluated an extended adjuvant treatment with neratinib after one year of trastuzumab

35. Martin $M$, Holmes FA, Eilertsen $B$, et al. Neratinib after trastuzumab-based adjuvant therapy in HER2-positive breast cancer (ExteNET): 5-year analysis of a randomised, double-blind, placebo-controlled, phase 3 trial. Lancet Oncol. 2017;18 (12):1688-1700.

36. Hurvitz S, Chan A, lannotti N, et al. Effects of adding budesonide or colestipol to loperamide prophylaxis on neratinib-associated diarrhea in patients with HER2+ early-stage breast cancer: the CONTROL trial [abstract]. Proceedings of the 2017 San Antonio Breast Cancer Symposium; 2017 Dec 5-9 San Antonio, TX. Philadelphia (PA): AACR Cancer Res 2018; 78(4Suppl):Abstract nr P3-14-01

37. http://www.ema.europa.eu/docs/en_GB/document_library/ Summary_of_opinion_-_Initial_authorisation/human/004030/ WC500244282.pdf

38. Goss PE, Smith IE, O'Shaughnessy J, et al. Adjuvant lapatinib for women with early-stage HER2- positive breast cancer: a randomised, controlled, phase 3 trial. Lancet Oncol. 2013;14:88-96.

39. Piccart-Gebhart MJ, Holmes AP, Baselga J, et al. First results from the phase III ALTTO trial (BIG 2-06; NCCTG [Alliance] N063D) comparing one year of anti-HER2 therapy with lapatinib alone (L), trastuzumab alone $(T)$, their sequence $(T \rightarrow L)$, or their combination $(\mathrm{T}+\mathrm{L})$ in the adjuvant treatment of HER2-positive early breast cancer (EBC). J Clin Oncol. 2014;32(suppl):abstr LBA4.

40. Piccart-Gebhart M, Holmes E, Baselga J, et al. adjuvant lapatinib and trastuzumab for early human epidermal growth factor receptor 2-positive breast cancer: results from the randomized phase III adjuvant lapatinib and/or trastuzumab treatment optimization trial. J Clin Oncol. 2016;34:1034-1042.

41. Slamon DJ, Swain SM, Buyse $M$, et al. Primary results from BETH, a phase 3 controlled study of adjuvant chemotherapy and trastuzumab \pm bevacizumab in patients with HER2-positive, nodepositive or high-risk node-negative breast cancer. 2013 San Antonio Breast Cancer Symposium; December 11, 2013; San Antonio, TX.

42. von Minckwitz G, Procter M, de Azambuja E, et al. Adjuvant pertuzumab and trastuzumab in Early HER2-positive breast cancer. N Engl J Med. 2017;377(2):122-131.

- In this trial the use of dual anti-HER2 directed treatment with adjuvant trastuzumab and pertuzumab was investigated, after showing positive results in the neoadjuvant setting

43. A study of trastuzumab emtansine versus trastuzumab as adjuvant therapy in patients with HER2-positive breast cancer who have residual tumor in the breast or axillary lymph nodes following preoperative therapy (KATHERINE). NCT01772472.

44. A Study of Trastuzumab Emtansine (Kadcyla) Plus Pertuzumab (Perjeta) Following Anthracyclines in Comparison With Trastuzumab (Herceptin) Plus Pertuzumab and a Taxane Following Anthracyclines as Adjuvant Therapy in Participants With Operable HER2-Positive Primary Breast Cancer. NCT01966471.

45. Loibl S, Majewski I, Guarneri V, et al. PIK3CA mutations are associated with reduced pathological complete response rates in primary HER2-positive breast cancer: pooled analysis of 967 patients from five prospective trials investigating lapatinib and trastuzumab. Ann Oncol. 2016;27:1519-1525.

46. Denkert C, von Minckwitz G, Darb-Esfahani S, et al. Evaluation of tumor-infiltrating lymphocytes (TILs) as predictive and prognostic biomarkers in different subtypes of breast cancer treated with neoadjuvant therapy-a meta-analysis of 3771 patients. Cancer Res. 2017;77(suppl):abstr S1-09. 
47. Perez EA, Ballman KV, Tenner KS, et al. Association of stromal tumor infiltrating lymphocytes with recurrence-free survival in the n9831 adjuvant trial in patients with early-stage HER2-positive breast cancer. JAMA Oncol. 2016;2:56-64.

48. Cortazar P, Zhang L, Untch $M$, et al. Pathological complete response and long-term clinical benefit in breast cancer: the CTNeoBC pooled analysis. Lancet. 2014;384:164-172.

49. Gianni L, Pienkowski T, Im YH, et al. 5-year analysis of neoadjuvant pertuzumab and trastuzumab in patients with locally advanced, inflammatory, or early-stage HER2-positive breast cancer (NeoSphere): a multicentre, open-label, phase 2 randomised trial. Lancet Oncol. 2016;17:791-800.

50. Schneeweiss A, Chia S, Hickish T, et al. Pertuzumab plus trastuzumab in combination with standard neoadjuvant anthracycline containing and anthracycline-free chemotherapy regimens in patients with HER2- positive early breast cancer: a randomized phase II cardiac safety study (TRYPHAENA). Ann Oncol. 2013;24:2278-2284.

51. Untch $M$, Jackisch $C$, Schneeweiss $A$, et al. Nab-paclitaxel versus solvent-based paclitaxel in neoadjuvant chemotherapy for early breast cancer (GeparSepto-GBG 69): a randomised, phase 3 trial. Lancet Oncol. 2016;17:345-356.

52. Llombart-Cussac A, Cortés J, Paré L, et al. HER2-enriched subtype as a predictor of pathological complete response following trastuzumab and lapatinib without chemotherapy in early-stage HER2-positive breast cancer (PAMELA): an open-label, single-group, multicentre, phase 2 trial. Lancet Oncol. 2017;18:545-554.

53. Rimawi MF, Mayer IA, Forero A, et al. Multicenter phase II study of neoadjuvant lapatinib and trastuzumab with hormonal therapy and without chemotherapy in patients with human epidermal growth factor receptor 2-overexpressing breast cancer: TBCRC 006. J Clin Oncol. 2013;31:1726-1731.

54. Rimawi MF, Niravath PA, Wang T, et al. TBCRC023: A randomized multicenter phase II neoadjuvant trial of lapatinib plus trastuzumab, with endcorine therapy and without chemotherapy, for 12 vs. 24 weeks in patients with HER2 overexpressing breast cancer. Cancer Res. 2015;75(9S):S602(SABCS).

55. Nitz UA, Gluz O, Christgen M, et al. De-escalation strategies in HER2positive early breast cancer (EBC): final analysis of the WSG-ADAPT HER2+/HR- phase II trial: efficacy, safety, and predictive markers for 12 weeks of neoadjuvant dual blockade with trastuzumab and pertuzumab \pm weekly paclitaxel. Ann Oncol. 2017;28:2768-2772.

56. Hurvitz SA, Martin M, Symmans WF, et al. Neoadjuvant trastuzumab, pertuzumab, and chemotherapy versus trastuzumab emtansine plus pertuzumab in patients with HER2-positive breast cancer (KRISTINE): a randomised, open-label, multicentre, phase 3 trial. Lancet Oncol. 2018;19:115-126. 